\title{
A peer victimisation scale based on a behavioural consequences measurement strategy
}

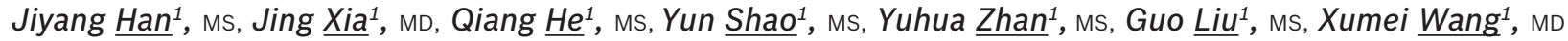

INTRODUCTION An accurate assessment of peer victimisation (i.e. bullying) is a necessary precondition for research and intervention. Most assessment instruments use the 'list of acts' measurement strategy, which does not account for the actual physical and psychological damage inflicted by bullying. To resolve this limitation, this study developed a peer victimisation scale (PVS) that includes harmful consequences for judgement and measurement of peer victimisation.

METHODS The PVS is a 40-item self-report questionnaire designed to assess the four aspects of peer victimisation: physical, verbal, relational, and interference and control. A total of 1,469 Grade 3-8 students (49.9\% male) were recruited to test the psychometric properties of the PVS. Another 420 Grade 3-8 students were examined by a modified PVS supplemented with a semi-structured interview for scale validation and establishment of the cut-off points for severe bullying. Incidence, age and gender distribution of peer victimisation were also analysed.

RESULTS The PVS demonstrated good internal consistency reliability (Cronbach's alpha 0.73-0.83) and test-retest reliability two weeks later (correlation coefficient $[r]=0.71-0.80)$. The scores for each dimension were significantly and positively correlated with the scores from the questionnaire-interview sample $(r=0.73-0.78)$, and modestly correlated with the scores for symptoms of anxiety and depression $(r=0.36-0.54)$.

CONCLUSION The results were consistent with the measurement constructs, demonstrating that the PVS is a reliable and effective instrument for assessing peer victimisation in children. It may enable more reliable longitudinal studies assessing the impact of peer victimisation to be conducted.

Keywords: assessment, children, peer victimisation, psychometrics, self-report

\section{INTRODUCTION}

Peer victimisation (i.e. bullying) among schoolchildren can result in lifelong psychiatric dysfunction. Many studies have demonstrated that bullied children experience more somatic symptoms, psychiatric disorders and social adjustment problems than children who have not been bullied. ${ }^{(1-3)}$ Bullying is a type of aggressive behaviour that is characterised by recurrence and a power imbalance. ${ }^{(1)}$ Aggressive behaviour has been defined as behaviour or speech that intends to harm others. ${ }^{(4)}$ This definition emphasises motivation, namely the intent to harm, along with the harmful characteristics of the act itself, but does not include harmful consequences as a necessary precondition to show victimisation. Longitudinal studies have shown that childhood/ adolescent bullying impairs adult psychosocial function and can result in persistent depressive symptoms, low self-esteem ${ }^{(5)}$ and difficulties in relating to the opposite gender. ${ }^{(6)}$ Intervention programmes for victims of peer victimisation (e.g. case-based intervention, change of school climate and strengthening of oversight) have been widely implemented and achieved encouraging results. ${ }^{(7)}$ In a number of countries, policies have been developed at the regional or national level to ensure the implementation of measures to reduce violence and bullying in schools. ${ }^{(8)}$

The value of studies assessing the impact of bullying on current and future psychological health depends on whether they have a precise definition of peer victimisation, as well as accurate metrics for the degree of bullying and the types of bullying experienced. Based on earlier studies, ${ }^{(9)}$ currently available instruments generally assess if respondents fall into the category of 'bullying others' or 'bullied by others' through self-report questionnaires that probe the frequency of items in a specific 'list of acts'. ${ }^{(10)}$ While these instruments may differ in their subscales, content and item wording, most adopt the same conceptual framework and operational definition. The 'list of acts' assessment is essentially a measurement of frequency, based on item choices. Discrepancies observed between different studies that use this form of assessment ${ }^{(11)}$ may be due to the fact that this method makes it difficult to establish standard thresholds or ranges to reflect 'mild', 'moderate' or 'severe' bullying behaviour or victimisation. Furthermore, even if there is repeated bullying behaviour at a particular frequency, it may not meet the criteria for harmful peer victimisation. ${ }^{(12)}$ It is also difficult to determine what should be included in these 'lists of acts' assessments.

Instruments developed according to the 'list of acts' strategy may be described as an item cohort of cause indicators. ${ }^{(13)}$ Each item that receives a positive answer may be translated to mean that a child has been 'bullied by others', but the intensity of the effects may vary; this is similar to the Social Readjustment Rating Scale, which quantifies stress according to the occurrence of certain life events. ${ }^{(14)}$ An instrument that assigns points to each bullying circumstance according to the intensity of the effect is a necessary prerequisite to ensure appropriate measurement scores. However, it is almost impossible to develop such an instrument, given that there are a multitude of possible bullying behaviours

${ }^{1}$ Department of Clinical Psychology, Shengjing Hospital of China Medical University, Liaoning, China

Correspondence: Prof Xumei Wang, Chief, Department of Clinical Psychology, Shengjing Hospital of China Medical University, No. 36 Sanhao Street, Heping District, Shenyang 110817, Liaoning, China. wangxumeimedsci@163.com 
and individual differences in sensitivity to these acts. To resolve this particular limitation, we developed a peer victimisation scale (PVS) that includes harmful consequences for judgement and measurement of peer victimisation. The aim of the present study was to evaluate the PVS' internal consistency reliability, test-retest reliability (after two weeks), and correlations with scores measuring symptoms of anxiety and depression in children and adolescents.

\section{METHODS}

This report describes the development of the PVS and the three stages of PVS validation (Fig. 1). PVS items were determined based on the responses collected via an open-ended questionnaire that was distributed to 2,138 middle school students, and the findings of on-site case interviews conducted among 317 children and adolescents.

The PVS is a self-report questionnaire for children and adolescents. The conceptual framework includes key points from the traditional definition of bullying, such as 'harmful intent', 'imbalance of power' and 'repeated behaviour', in addition to a new judgement criteria, 'harmful consequences'. Each item was devised to describe a circumstance that can reflect both the behavioural traits and harmful consequences of bullying, from direct acute physical and emotional experiences (e.g. injury, hurting and humiliation) to chronic effects on mood or disposition (e.g. feeling insecure in school), and secondary behaviours (e.g. avoidance of certain places or people) (Fig. 2).
Harmful consequences can be used as a basis to differentiate peer victimisation from benign childhood behaviour if there is a causal link between harmful consequences and peer victimisation. Bullying-related consequences that were more general (e.g. somatisation and poor academic performance) were not included as questionnaire items because their associations with specific instances of bullying are indirect and difficult to represent using single-item criteria. The PVS measures four aspects of peer victimisation: physical victimisation, verbal victimisation, relational victimisation, and interference and control. The severity of a particular type of bullying is reflected by the subscore. This measurement strategy provides a basis for interpreting measurement scores, thereby yielding effective measurement of peer victimisation.

This study used the following two approaches to describe peer victimisation: (a) an open-ended questionnaire; and (b) an on-site case interview. From September 2010 to November 2010, all students in Grades 3-9 from two elementary schools and four middle schools in Shenyang, China, were asked to complete the open-ended questionnaires, which sought to identify experiences of peer victimisation. A total of 2,138 questionnaires were returned; $52.5 \%$ were completed by male students. The questionnaire provided the operational definition of peer victimisation and had open-ended questions that allowed respondents to describe their experiences with peer victimisation (i.e. the circumstances and their feelings in response to the incidents). The answers were in the form of a few sentences

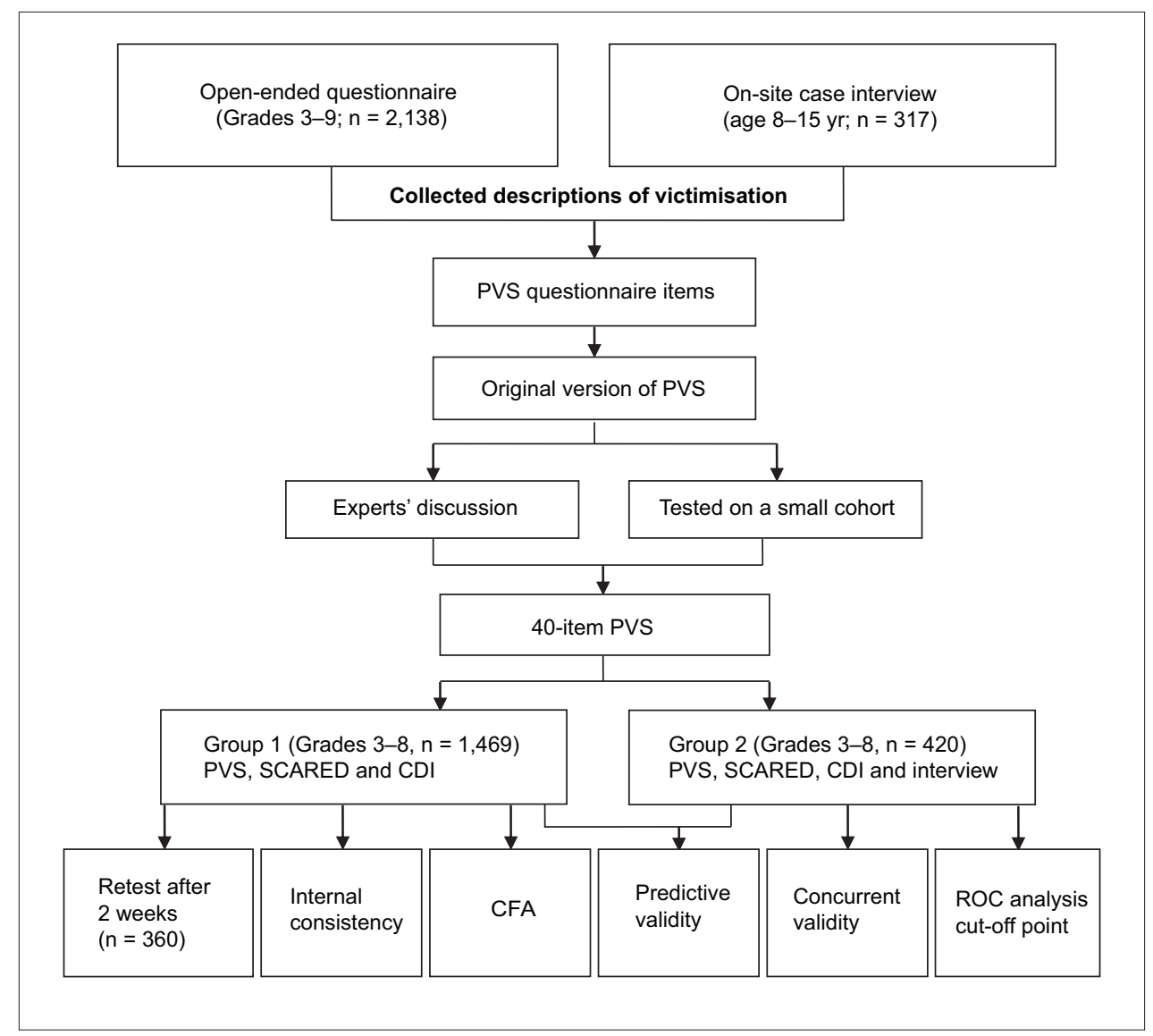

Fig. 1 Flowchart shows the study design. CDI: Children's Depression Inventory; CFA: confirmatory factor analysis; PVS: peer victimisation scale; ROC: receiver operating characteristic; SCARED: Screen for Child Anxiety-Related Emotional Disorders 


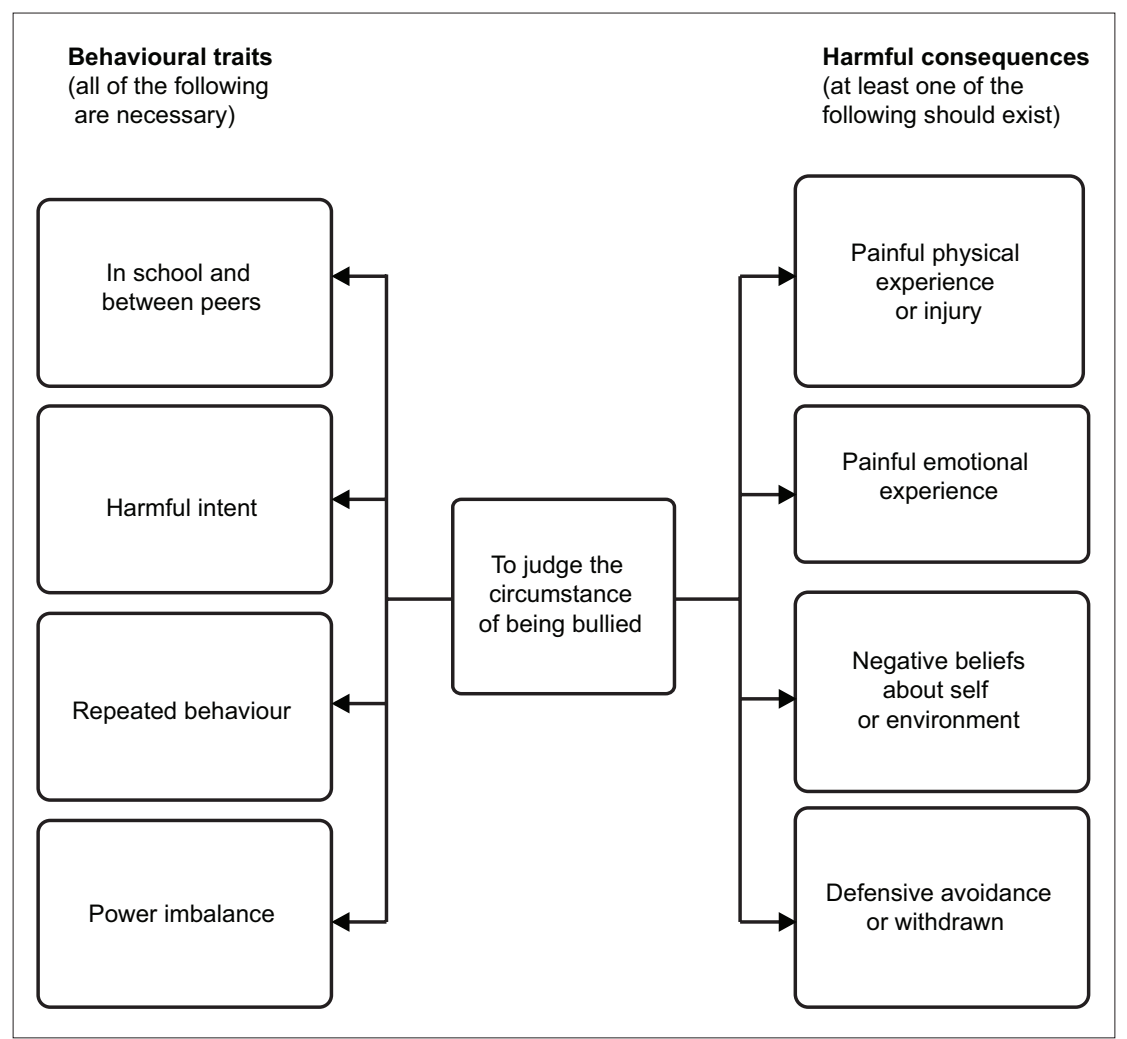

Fig. 2 Flowchart shows the measurement constructs of the peer victimisation scale (PVS). The PVS measures peer victimisation using behavioural traits and harmful consequences. In the present study, the PVS was used to measure peer victimisation in the academic setting.

and each student was allowed to include up to five different experiences. A total of 789 (36.9\%) questionnaires reported previous experiences of peer victimisation and a total of 1,746 descriptions were submitted.

From December 2008 to September 2010, we conducted onsite case interviews involving 317 children and adolescents (age 8-15 years, 50.8\% male) at the Psychological Clinic of Shengjing Hospital of China Medical University, China. The respondents' descriptions of their personal experiences or accounts of other people's experiences of peer victimisation, including the impact and corresponding circumstances, were recorded. Among these 317 students, 80 (25.2\%) reported that they were 'often bullied by others'.

The information collected was compiled and categorised. In this study, peer victimisation was categorised into four dimensions, based on previous studies: ${ }^{(10,12,14)}$ physical bullying, verbal bullying, relational bullying, and interference and control. The PVS questionnaire items focus mainly on judgement elements of peer victimisation, including harmful characteristics of the behaviour (e.g. Item 9: 'My classmates give me a terrible nickname'), intent to harm (e.g. Item 1: 'Some classmates deliberately and repeatedly ignore $\mathrm{me}^{\prime}$ ), imbalance of power (e.g. Item 35: 'I am unable to fight back when my classmates attack me'), repeated behaviour (e.g. Item 25: 'Some classmates always curse at me'), painful physical and emotional experiences (e.g. Item 15: 'Some classmates always deliberately hurt or injure me' and Item 11: 'I am upset because I lack friends'), peer victimisation-induced ideation (e.g. Item 8: 'I wish I could be stronger so that I can be free from bullies'), and secondary behaviours (e.g. Item 37: 'I do everything carefully to avoid being laughed at by my classmates').

A team of experts discussed the scale draft based on: (a) the correlation between the items and measurement goals; (b) the clarity and accuracy of the language; (c) the possibility of inducing dislike of tested subjects in the instructions or item descriptions; and (d) the addition or deletion of items. The scale draft was tested on a small cohort of children for content validity and clarity, and to ensure that the children were able to read and understand the items. Several expressions were adjusted based on the reading ability of third-graders.

The final version of the PVS included 40 items that covered the four dimensions of peer victimisation: physical victimisation (ten items), verbal victimisation (ten items), relational victimisation (11 items), and interference and control (nine items). Each item asked children to describe their experience of bullying-related events in the past three months; responses were scored from $0-3(0=$ never or barely; $1=$ sometimes; $2=$ frequently; and $3=$ all the time). The questionnaire instructions defined 'other kids' as students from all classes and grades in the school. Inverse items were not included in the PVS and the scores for each dimension (i.e. the subscores) were obtained by adding up the points of corresponding items.

The PVS was validated by examining a large test group of middle school students (Group 1) and a smaller group of students who completed both the PVS and a semi-structured interview in a clinical setting (Group 2). In addition, both Groups 1 and 2 completed the Screen for Child Anxiety-Related Emotional Disorders (SCARED) questionnaire and the Children's 
Depression Inventory (CDI). Selected students from Group 1 were retested two weeks later.

Group 1 consisted of a group of students recruited from six typical schools (three elementary schools and three middle schools) in metropolitan Shenyang, China. None of the schools or students had been involved in the previous questionnaire. All students in Grades 3-8 were included. A total of 1,551 questionnaires including the PVS, SCARED and CDI were distributed to the students and 1,469 (94.7\%) valid questionnaires were returned. 82 (5.3\%) questionnaires were excluded from the subsequent analyses because they were defaced and/or were missing more than two items. The survey was conducted in a classroom and monitored by one teacher and one researcher. It took approximately 20-30 minutes to complete the entire questionnaire. Of the 1,469 valid questionnaires (49.9\% male), 198 came from third-graders, 462 from fourth-graders, 276 from fifth-graders, 212 from sixth-graders, 151 from seventh-graders, and 170 from eighth-graders. Students from two classes from each grade were randomly selected for retesting two weeks later, under the same survey conditions. Among the students selected for retesting, 360 filled out valid questionnaires both times (50.3\% male) - 63 were third-graders, 63 were fourth-graders, 67 were fifth-graders, 59 were sixth-graders, 54 were seventh-graders and 54 were eighth-graders.

Group 2 consisted of 420 paediatric patients ( $47.1 \%$ male) who were tested at the Department of Paediatrics, Shengjing Hospital of China Medical University, China, from September 2011 to February 2012. Patients who met the following criteria were eligible for inclusion: (a) both the patients and their guardians agreed to participate; (b) were in Grades 3-8; (c) lived in Liaoning province, China; and (d) were currently schooling. Among the 420 patients who met the criteria, 74 were third-graders, 72 were fourth-graders, 58 were fifth-graders, 71 were sixth-graders, 76 were seventh-graders and 69 were eighth-graders. Students who could not attend school due to illness and those who were unable to collaborate with the interviewer due to physical limitations were excluded. Among these 420 patients, $58.8 \%$ resided in large or middle-sized cities, $25.2 \%$ in townships and $16.0 \%$ in rural areas. All the respondents completed a self-report questionnaire, consisting of the PVS, CDI and SCARED, in the waiting room before being interviewed.

The 5-20-minute, semi-structured interview was conducted in an examination room by two researchers in the presence of the child's guardian. The goal was to understand each child's experience of peer victimisation during the previous three months. The PVS questionnaire was used as the outline for the interview. Physical victimisation included kicking, beating, pushing, throwing objects, pinching, slapping and hair pulling. Verbal bullying included scolding, attacks on defects or weaknesses, name-calling, laughing at others and use of nasty words. Relational bullying included spreading rumours, ignoring and/or rejecting others, distancing one's self from others, and obstructing interactions between others and the bullied child. Acts of interference and control included interfering with the bullied child's studies or other activities, bluffing, mimicking the bullied child's speech or acts, coercion, grabbing the bullied child's personal belongings, hiding or damaging items, and committing pranks. The key elements in the judgement of peer victimisation and the severity of victimisation were frequency, imbalance of power among the individuals involved, and pain and distress caused by bullying. Based on the interview, the interviewer scored each category of bullying on the following four-point scale: $0=$ no bullying or mild bullying that did not cause pain or distress; 1 = bullying was present, but it was not serious and resulted in only slight pain or distress; 2 = serious bullying that caused the victim significant pain or distress, but did not impair the victim's ability to continue with his/her studies and interact with others; and 3 = severe bullying that caused the victim unbearable pain or distress, such that the victim was unable to continue his/her studies or interact with others. After the interview, the two researchers discussed and filled out the interview questionnaires for peer victimisation. The researchers were not allowed to view the students' self-report questionnaires before they completed the interview and scoring.

The CDI, which was developed by Maria Kovacs, ${ }^{(15)}$ is a 27-item self-report inventory of childhood depression. Each item consists of three sentences (describing certain circumstances that occurred during the previous two weeks) that represent three levels of depression (scored as 0,1 or 2 points). The CDI is suitable for use among children aged 7-17 years. The total CDI score ranges from 0-54 points, with higher scores indicating a higher level of depression. The Chinese version of the CDI, which was developed and amended by David and Li in 2000, ${ }^{(16)}$ demonstrated good reliability and validity in preliminary tests (Group 1: Cronbach's alpha 0.91).

The SCARED questionnaire, developed by Birmaher et al, ${ }^{(17)}$ is a self-report assessment tool for diagnosing anxiety disorders in children aged 9-18 years. The original scale had 38 items. Each item describes one anxiety-related manifestation and requires the respondent to answer a question based on his/her experience during the previous three months. Items are scored on a threepoint scale $(0=$ not true or hardly ever true; $1=$ sometimes true; and $2=$ true or often true). The total score is the sum of the points for all 38 items and higher scores indicate that the child is more likely to have an anxiety disorder. The SCARED inventory was amended by Su et al in 2008 ${ }^{(18)}$ to include 41 items (Group 1: Cronbach's alpha 0.92).

All respondents and their guardians were informed about the on-site interview and written informed consent was obtained. The questionnaire used at the schools was approved by the person in charge of each school, who signed the informed consent forms and confidentiality agreements. The questionnaire was completed anonymously (with the exception of the retested students) and there were clear, printed instructions on the questionnaire informing respondents that they could refuse to complete it without any penalty. The overall research plan was approved by the Ethics Committee of Shengjing Hospital of China Medical University, China.

The internal consistency reliability of the PVS was evaluated using the corrected item-total correlation and Cronbach's alpha 
tests. Pearson's correlation coefficient was used to analyse the test-retest reliability, relationship between PVS dimension and total scores of both the CDI and SCARED questionnaires, and consistency between the PVS dimension scores and responses from the semi-structured interviews. The cut-off values were determined using a receiver operating characteristic (ROC) curve. Peer victimisation rates were compared between the two genders and the different age groups using Pearson's chi-square test. Confirmatory factor analysis (CFA) to analyse the factorial validity of the PVS was performed using SPSS version 7.0 (SPSS Inc, Chicago, IL, USA). All other analyses were performed using SPSS version 16.0 (SPSS Inc, Chicago, IL, USA). A p-value $<0.05$ was considered statistically significant.

\section{RESULTS}

Item analysis was performed to evaluate the contribution of each item to its corresponding dimension. The results were used to test the effectiveness of the internal structure of the PVS. We calculated the correlation between each corrected item and the result of each total dimension score minus that item score. Only four items (i.e. Items 8, 21, 29 and 37) had correlation coefficients $<0.3$ (Table I). All total dimension scores were moderately correlated, with correlation coefficients $0.65-0.70$ $(\mathrm{n}=1,469 ; \mathrm{p}<0.001)$.

The test-retest reliability of the respective PVS dimensions were as follows: 0.73 for physical victimisation, 0.75 for verbal victimisation, 0.80 for relational victimisation, and 0.71 for interference and control $(n=360 ; p<0.001)$. Cronbach's alpha test was used to calculate the internal consistency reliability for each dimension. In Group 1, Cronbach's alpha was 0.77 for physical victimisation, 0.77 for verbal victimisation, 0.83 for relational victimisation, and 0.73 for interference and control. In Group 2, Cronbach's alpha was 0.77 for physical victimisation, 0.79 for verbal victimisation, 0.83 for relational victimisation, and 0.74 for interference and control. All the Cronbach's alpha coefficients were $>0.7$, suggesting that all the PVS dimensions had acceptable internal consistency reliability.

Concurrent validity is a validity criterion used to confirm the correlation of a measure with external empirical evidence. The scores of the interview questionnaire on peer victimisation were used as the criteria to calculate the correlation coefficient for each corresponding PVS dimension. The validity coefficients for each dimension were as follows: 0.73 for physical victimisation, 0.78 for verbal victimisation, 0.76 for relational victimisation, and 0.74 for interference and control $(n=420, p<0.001)$.

Correlation analyses of the CDI and SCARED total scores versus each PVS dimension score were used to test for the presence of logical relationships. The correlation coefficients ranged from 0.36-0.54, indicating poor correlation but nevertheless suggesting possible cause-and-effect relationships (Table II).

Exploratory factor analysis (EFA) was carried out, but did not yield understandable or logical results. Thus, SPSS version 7.0 was used to conduct CFA of the PVS dimensions. Maximum likelihood estimation was used to analyse the goodness of fit for the four-factor model. As the rate of missing data was less
Table I. Results of the item analysis to determine corrected item and total dimension score correlation $(n=1,469)$.

\begin{tabular}{|c|c|c|c|c|c|c|c|}
\hline \multicolumn{2}{|c|}{$\begin{array}{c}\text { Physical } \\
\text { victimisation }\end{array}$} & \multicolumn{2}{|c|}{$\begin{array}{c}\text { Verbal } \\
\text { victimisation }\end{array}$} & \multicolumn{2}{|c|}{$\begin{array}{c}\text { Relational } \\
\text { victimisation }\end{array}$} & \multicolumn{2}{|c|}{$\begin{array}{l}\text { Interference } \\
\text { and control }\end{array}$} \\
\hline Item & $\mathbf{r}$ & Item & $\mathbf{r}$ & Item & $\mathbf{r}$ & Item & $\mathbf{r}$ \\
\hline 8 & $0.26^{*}$ & 2 & 0.47 & 1 & 0.50 & 3 & 0.32 \\
\hline 15 & 0.43 & 9 & 0.43 & 4 & 0.51 & 6 & 0.42 \\
\hline 18 & 0.51 & 12 & 0.34 & 5 & 0.56 & 7 & 0.44 \\
\hline 20 & 0.46 & 13 & 0.54 & 11 & 0.44 & 10 & 0.47 \\
\hline 27 & 0.43 & 14 & 0.44 & 22 & 0.55 & 17 & 0.52 \\
\hline 30 & 0.49 & 16 & 0.59 & 23 & 0.62 & 19 & 0.50 \\
\hline 31 & 0.50 & 25 & 0.53 & 24 & 0.37 & 21 & 0.24 * \\
\hline 34 & 0.51 & 37 & $0.26^{*}$ & 28 & 0.54 & 26 & 0.42 \\
\hline 35 & 0.46 & 38 & 0.42 & 29 & $0.27^{*}$ & 32 & 0.45 \\
\hline \multirow[t]{2}{*}{36} & 0.48 & 39 & 0.53 & 33 & 0.55 & & \\
\hline & & & & 40 & 0.58 & & \\
\hline
\end{tabular}

All p-values were $<0.001 .{ }^{*} r<0.3 . r$ : correlation coefficient

Table II. Correlation coefficients of the Children's Depression Inventory (CDI) and Screen for Child Anxiety-Related Emotional Disorders (SCARED) total scores versus the score of each Peer Victimisation Scale (PVS) dimension.

\begin{tabular}{|c|c|c|c|c|c|c|c|c|}
\hline \multirow[t]{3}{*}{ Variable } & \multicolumn{8}{|c|}{ PVS dimension } \\
\hline & \multicolumn{4}{|c|}{ Group $1(n=1,469)$} & \multicolumn{4}{|c|}{ Group $2(n=420)$} \\
\hline & PV & VV & RV & IC & PV & VV & RV & IC \\
\hline CDI & 0.54 & 0.46 & 0.53 & 0.43 & 0.50 & 0.44 & 0.46 & 0.42 \\
\hline SCARED & 0.47 & 0.44 & 0.43 & 0.37 & 0.49 & 0.42 & 0.40 & 0.36 \\
\hline
\end{tabular}

All p-values were < 0.001. IC: interference and control; PV: physical victimisation; $\mathrm{RV}$ : relational victimisation; VV: verbal victimisation

than $1.0 \%$, the missing numbers were replaced by the average subscore. The goodness of fit for the four-factor model and the results of Group 1 were as follows: $\chi^{2}=3738.853$; degrees of freedom $(\mathrm{df})=734 ; \mathrm{p}<0.001 ; \chi^{2} / \mathrm{df}=5.094 ;$ goodness-of-fit index $(\mathrm{GFI})=0.874$; adjusted GFI $($ AGFI $)=0.859$; comparative fit index $=0.828$; Tucker-Lewis index $=0.817$; root mean square error of approximation $=0.053$; and root mean square residual $=0.030$. Some GFIs did not fulfil the measurement criteria; after the model was modified with methods related to the residuals, the GFIs and other indices fulfilled the measurement criteria.

A score of $\geq 2$ points in each category of the interview questionnaire on peer victimisation (i.e. indicating serious or severe bullying) was used as the reference criteria for evaluating peer victimisation and for graphing the ROC curve of the score for each PVS dimension. The area under the ROC curve, and the sensitivity and specificity of each threshold were calculated (Table III). The maximum Youden index (sensitivity + specificity -1 ) was used as the reference cut-off point. The reference cut-off points for the four dimensions were as follows: 12 points for physical victimisation; 15 points for verbal victimisation; 13 points for relational victimisation; and 10 points for interference and control.

Based on the aforementioned cut-off points, analysis was conducted to assess the prevalence of peer victimisation within Group 1 ( $n=1,469)$. The number of students who experienced 
Table III. Sensitivity and specificity of the corresponding cut-off points for each peer victimisation scale (PVS) dimension ( $\mathbf{n}=420)$.

\begin{tabular}{|c|c|c|c|c|c|c|c|c|}
\hline \multirow{3}{*}{$\begin{array}{l}\text { Cut-off } \\
\text { point }\end{array}$} & \multicolumn{8}{|c|}{ PVS dimension } \\
\hline & \multicolumn{2}{|c|}{ PV (AUC = 0.967) } & \multicolumn{2}{|c|}{ VV $(A \cup C=0.920)$} & \multicolumn{2}{|c|}{ RV (AUC = 0.955) } & \multicolumn{2}{|c|}{ IC (AUC = 0.963) } \\
\hline & SEN & SPE & SEN & SPE & SEN & SPE & SEN & SPE \\
\hline 7.5 & 0.864 & 0.947 & 0.900 & 0.856 & 0.920 & 0.919 & 0.846 & 0.964 \\
\hline 8.5 & 0.818 & 0.965 & 0.900 & 0.910 & 0.880 & 0.947 & 0.846 & 0.985 \\
\hline 9.5 & 0.818 & 0.977 & 0.867 & 0.936 & 0.880 & 0.957 & $0.846^{*}$ & $0.995^{*}$ \\
\hline 11.5 & $0.818^{*}$ & $0.995^{*}$ & 0.867 & 0.964 & 0.880 & 0.982 & 0.577 & 0.997 \\
\hline 12.5 & 0.682 & 0.995 & 0.867 & 0.977 & $0.880^{*}$ & $0.997^{*}$ & 0.385 & 0.997 \\
\hline 13.5 & 0.636 & 0.997 & 0.867 & 0.987 & 0.720 & 1.000 & 0.308 & 1.000 \\
\hline 14.5 & 0.455 & 1.000 & $0.867^{*}$ & $0.995^{*}$ & 0.600 & 1.000 & 0.269 & 1.000 \\
\hline 15.5 & 0.318 & 1.000 & 0.600 & 0.997 & 0.520 & 1.000 & 0.154 & 1.000 \\
\hline 16.5 & 0.273 & 1.000 & 0.533 & 1.000 & 0.440 & 1.000 & 0.115 & 1.000 \\
\hline
\end{tabular}

For the selected cut-off values, the positive and negative predictive values were: PV: 0.900 and 0.990; VV: 0.929 and $0.990 ; \mathrm{RV}: 0.957$ and 0.992 ; and IC: 0.917 and 0.990 , respectively. ${ }^{*}$ Maximum Youden index. AUC: area under the curve; IC: interference and control; PV: physical victimisation; RV: relational victimisation; SEN: sensitivity; SPE: specificity; VV: verbal victimisation

Table IV. Comparison of the prevalence of peer victimisation, according to age group and gender.

\begin{tabular}{|c|c|c|c|c|c|c|}
\hline \multirow[t]{3}{*}{ Variable } & \multicolumn{6}{|c|}{$\%$} \\
\hline & \multicolumn{3}{|c|}{ Elementary school } & \multicolumn{3}{|c|}{ Middle school } \\
\hline & Male $(n=564)$ & Female $(n=584)$ & All $(n=1,148)$ & Male $(n=169)$ & Female $(n=152)$ & All $(n=321)$ \\
\hline PV & 9.6 & $3.6^{+t}$ & 6.5 & $4.1^{\neq}$ & 2.6 & $3.4^{\neq}$ \\
\hline VV & 8.5 & $4.8^{+}$ & 6.6 & 5.3 & 3.9 & 4.7 \\
\hline $\mathrm{RV}$ & 8.9 & $5.8^{+}$ & 7.2 & $3.0^{\ddagger}$ & 6.6 & 4.7 \\
\hline Any* & 19.0 & $11.1^{t+}$ & 15.0 & $10.1^{\neq \neq}$ & 9.2 & $9.7^{\ddagger}$ \\
\hline
\end{tabular}

*Victims of at least one category of peer victimisation. ${ }^{t} p<0.05$ for male vs. female students within the same school level. ${ }^{+t p}<0.01$ for male vs. female students within the same school level. $\neq p<0.05$ for elementary school (Grades $3-6$ ) vs. middle school students (Grades $7-8$ ) for the same gender or for all students. $\neq \equiv p<0.01$ for elementary school vs. middle school students for the same gender or for all students. IC: interference and control; PV: physical victimisation; RV: relational victimisation; VV: verbal victimisation

the different categories of peer victimisation was as follows: 86 (5.9\%) suffered from physical bullying, 91 (6.2\%) suffered from verbal bullying, 98 (6.7\%) suffered from relational bullying, and $91(6.2 \%)$ suffered from interference and control. A total of $203(13.8 \%)$ students were the victims of at least one category of bullying. The prevalence of male students in all categories of peer victimisation was higher than that of female students $(p<0.05)$ in elementary school; no gender differences in the prevalence of peer victimisation was found among the students in middle school. Among the male students, the frequencies of physical victimisation, relational victimisation, and interference and control were higher among those in elementary school than among those in middle school $(p<0.05)$. Among the female students, there were no significant differences in the frequencies of the four peer victimisation categories between those in elementary school and those in middle school (Table IV).

\section{DISCUSSION}

In contrast with other peer victimisation instruments that focused on the frequency of bullying and did not take into account the impact of bullying on the victim, the PVS we developed included 'harmful consequences' as a criterion for identifying and measuring peer victimisation. The inclusion of key concepts from traditional definitions of bullying (including 'harmful intent', 'imbalance of power' and 'repeated behaviour' $)^{(1)}$ allows the PVS results to be directly compared with the results of previous studies that utilise other peer victimisation instruments; furthermore, the inclusion of 'harmful consequences' may enhance the predictive validity of the PVS. We observed poor-to-moderate correlation between PVS scores and the scores obtained using the CDI and SCARED questionnaires for childhood depression and anxiety, respectively. In CFA, the GFI and AGFI were both close to 0.9, indicating that the PVS is an acceptable model. ${ }^{(19)}$ Thus, the PVS may be suitable for use in longitudinal studies that seek to examine long-term psychosocial function impairments among victims of childhood bullying.

Compared with instruments that are based on the 'list of acts' strategy, scales based on behavioural consequences measure the relationship between objective facts and subjective feelings. In addition, the cut-off values to relate composite scores to the severity of bullying were also established in the present study. We verified that each PVS dimension had acceptable internal consistency reliability (Cronbach's alpha $>0.7$ ) and test-retest reliability (retest was conducted two weeks after the first test). The correlation coefficient of each dimension's subscore was strongly correlated to those derived from the combined questionnaire and semi-structured interview (scores ranged from 0.73-0.78), suggesting good concurrent validity. Depression 
and anxiety are adjustment problems that are closely associated with peer victimisation. ${ }^{(11,20,21)}$ The score for each PVS dimension was moderately related to the CDI and SCARED total scores, consistent with the 'harmful consequences' measurement concept. These modest correlations suggest possible cause-andeffect relationships.

Bullying is often characterised by its method (e.g. physical, verbal, relational, sexual and cyber bullying); ${ }^{(11,22,23)}$ the PVS in the present study uses the three categories of physical, verbal and relational bullying, and added the category of interference and control. The harmful consequences of these four types of bullying are not the same. Physical bullying causes physical pain and a sense of insecurity; verbal bullying results in humiliation; relational bullying damages peer relationships; and interference and control leads to decreased autonomy. The PVS did not include dimensions related to sexual or cyber bullying because among the 1,746 descriptions of peer victimisation collected via our open-ended questionnaire during the development stage, only two were related to sexual bullying and four to cyber bullying. Among the 317 students who participated in the on-site case interview, only one student reported receiving a malicious text message and feeling upset. There were no case reports of sexual bullying identified during the on-site case interviews. Given the age range of the participants (Grades 3-8), the actual prevalence of sexual bullying was most likely low. Also, discussion of sexrelated topics is taboo in traditional Chinese culture, ${ }^{(24)}$ which may have reduced the likelihood of students reporting sexual bullying. During our school visits, we learned that the students had little free time and were not allowed to use mobile phones or access the Internet while in school, thereby reducing the prevalence of cyber bullying in school.

The results of the present study confirmed the universality of peer victimisation. Among the students from Group $1(n=1,469)$, $13.8 \%$ reported experiencing at least one of the four defined forms of peer victimisation, resulting in significant anxiety and depression. The distribution of school ages and grades was similar to that reported by Olweus. ${ }^{(25)}$ In the present study, we found a downward trend in the prevalence of bullying, beginning in the middle-to-upper grades of elementary school through middle school; this was mainly a reflection of the reduced prevalence of bullying by male students. At the upper grades of elementary school, the prevalence of bullying was significantly higher among male students than among female students, while there was no significant difference in the prevalence of bullying between the two genders in middle school. These results are similar to those reported by Nansel et $\mathrm{al}^{(1)}$ and Kim et al. ${ }^{(26)}$ We speculate that peer victimisation is most prominent among male students in the upper grades of elementary school. Among the categories of peer victimisation, we found that the incidences of physical victimisation, and interference and control were significantly lower in middle school than in elementary school, while no significant differences were found in the incidences of verbal and relational victimisation between these age groups.

There are numerous self-report instruments that measure bullying behaviour, victimisation, or both, in children and adolescents. Victimisation scales include the Gatehouse Bullying Scale (GBS), ${ }^{(27)}$ Multidimensional Peer Victimization Scale (MPVS), (28) 'My Life in School' checklist, ${ }^{(29)}$ comprehensive Retrospective Bullying Questionnaire, ${ }^{(30)}$ Perception of Teasing Scale, ${ }^{(31)}$ Orpinas victimisation scale, ${ }^{(32)}$ Adolescent Peer Relations Instrument ${ }^{(33)}$ and Weight-based Teasing Scale (a more focused instrument). ${ }^{(34)}$ Many of these instruments, such as the GBS, MPVS and 'My Life in School' checklist, document bullying according to the frequency of specific events, while others, such as the comprehensive Retrospective Bullying Questionnaire, are long-form instruments that are tailored for small cohorts and not convenient for large-group analysis. There are few comprehensive instruments that focus on the impact of bullying on victims, but none that are developed in the Chinese cultural context and validated for Chinese adolescents. Thus, our PVS provides a simple 40-item 'multiple-choice' format questionnaire that is suitable for large-scale surveys and for the measurement of the impact of peer victimisation on Chinese children and adolescents.

The present study was not without limitations. Our study only included urban students for both the development and testing of the PVS. Cultural differences between students from cities and rural areas may influence the frequency, type and impact of bullying. In addition, some of the goodness of fit values in the four-factor analysis did not fulfil the measurement criteria. Although the basis of our study was to investigate the harmful consequences of the different categories of peer victimisation, there were overlaps among these consequences. In addition, a single type of bullying could have and lead to several harmful consequences. Further refinement of this theoretical framework based on larger samples is required. Another limitation of the present study was that there was no similar instrument adapted to the Chinese population for comparison. Thus, we used a modified version combined with direct clinical assessments to test the validity of the PVS and establish cut-off scores for differentiating the different degrees of bullying. Such interviews are inevitably influenced by subjective factors. Finally, although we performed EFA, the explored factors did not provide any understandable and logical results in the EFA. Therefore, we could not determine any theoretical framework. Since the EFA is known to be more accurate when each factor is represented by multiple measured variables, it is possible that our EFA was not successful as certain factors did not have enough variables. We used an alternative method since the EFA was not successful, hypothesising the PVS dimension grouping according to guidelines and expert consensus. If the present study had been purely exploratory, the CFA would have been the wrong approach. However, since the literature allowed the present study to be built on valid hypotheses, the CFA approach was used. ${ }^{(35,36)}$

To conclude, the PVS is a new measurement strategy for the assessment of peer victimisation, with acceptable internal consistency reliability, consistent test-retest reliability, and predictive validity for signs of childhood anxiety and depression related to bullying. In the PVS, 'harmful consequences' was introduced as a key element in the judgement of peer victimisation. Each PVS dimension displayed good homogeneity and reliability, 
and the evidence of concurrent validity was consistent with the measurement concept. The present study confirms previous research on the popularity of peer victimisation in China and elsewhere, ${ }^{(1,11,26)}$ and may have the additional advantage of taking into account the influence of bullying on both current and future psychopathology. We have plans to conduct additional research to confirm the predictive value of the PVS.

\section{REFERENCES}

1. Nansel TR, Overpeck M, Pilla RS, et al. Bullying behaviors among US youth: prevalence and association with psychosocial adjustment. JAMA 2001; 285:2094-100.

2. Nansel TR, Craig W, Overpeck MD, Saluja G, Ruan WJ; Health Behaviour in School-aged Children Bullying Analyses Working Group. Crossnational consistency in the relationship between bullying behaviors and psychosocial adjustment. Arch Pediatr Adolesc Med 2004; 158:730-6.

3. Gini G. Associations between bullying behaviour, psychosomatic complaints, emotional and behavioural problems. J Paediatr Child Health 2008; 44:492-7.

4. Brown JD. Social psychology. New York: McGraw-Hill, 2006.

5. Olweus D. Victimisation by peers: antecedents and long-term outcomes. In: Rubin KH, Asendorf JB, eds. Social withdrawal, inhibition, and shyness. Hillsdale, NJ: Erlbaum, 1993: 315-41.

6. Gilmartin BG. Peer group antecedents of severe love-shyness in males. J Pers 1987; 55:467-89.

7. Vreeman RC, Carroll AE. A systematic review of school-based interventions to prevent bullying. Arch Pediatr Adolesc Med 2007; 161:78-88.

8. Midthassel UV, Minton SJ, O'Moore AM. Conditions for the implementation of anti-bullying programmes in Norway and Ireland: a comparison of contexts and strategies. Compare 2009; 39:737-50.

9. Olweus D. Aggression in the schools: bullies and whipping boys. Washington, DC: Hemisphere Publishing Corp, 1978.

10. Felix ED, Sharkey JD, Green JG, Furlong MJ, Tanigawa D. Getting precise and pragmatic about the assessment of bullying: the development of the California Bullying Victimisation Scale. Aggress Behav 2011; 37:234-47.

11. Hawker DS, Boulton MJ. Twenty years' research on peer victimisation and psychosocial maladjustment: a meta-analytic review of cross-sectional studies. J Child Psychol Psychiatry 2000; 41:441-55.

12. Naylor $P$, Cowie H, Cossin F, de Bettencourt R, Lemme F. Teachers' and pupils' definitions of bullying. Br J Educ Psychol 2006; 76(Pt 3):553-76.

13. DeVellis RF. Scale development: theory and applications. 2nd ed. Thousand Oaks, CA: Sage Publications, 2003.

14. Holmes TH, Rahe RH. The Social Readjustment Rating Scale. J Psychosom Res 1967; 11:213-8.

15. Timbremont B, Braet C, Dreessen L. Assessing depression in youth: relation between the Children's Depression Inventory and a structured interview. J Clin Child Adolesc Psychol 2004; 33:149-57.

16. David Y, Li X. [Preliminary use of the Children's Depression Inventory in China]. Zhongguo Xin Li Wei Sheng Za Zhi 2000; 14:225-7. Chinese.

17. Birmaher B, Khetarpal S, Brent D, et al. The Screen for Child Anxiety Related Emotional Disorders (SCARED): scale construction and psychometric characteristics. J Am Acad Child Adolesc Psychiatry 1997; 36:545-53.
18. Su L, Wang K, Fan F, Su Y, Gao X. Reliability and validity of the screen for child anxiety related emotional disorders (SCARED) in Chinese children. J Anxiety Disord 2008; 22:612-21.

19. Baumgartner $\mathrm{H}$, Homburg $\mathrm{C}$. Applications of structural equation modeling in marketing and consumer research: A review. Int J Res Market 1996; 13:139-61.

20. Kaltiala-Heino R, Rimpelä M, Marttunen M, Rimpelä A, Rantanen P. Bullying, depression, and suicidal ideation in Finnish adolescents: school survey. BMJ 1999; 319:348-51.

21. Kumpulainen K, Räsänen E, Henttonen I, et al. Bullying and psychiatric symptoms among elementary school-age children. Child Abuse Negl 1998; 22:705-17.

22. Lamb J, Pepler DJ, Craig W. Approach to bullying and victimisation. Can Fam Physician 2009; 55:356-60.

23. Vieno A, Gini G, Santinello M. Different forms of bullying and their association to smoking and drinking behavior in Italian adolescents. J Sch Health $2011 ; 81: 393-9$.

24. Liu H, Feng T, Ha T, et al. Chinese Culture, Homosexuality Stigma, Social Support and Condom Use: A Path Analytic Model. Stigma Res Action 2011; $1: 27-35$

25. Olweus D. Bully/victim problems among schoolchildren: basic facts and effects of a school based intervention program. In: Rubin K, Pepler DJ, eds. The development and treatment of childhood aggression. Hillsdale, NJ: Erlbaum, 1991.

26. Kim YS, Koh YJ, Leventhal BL. Prevalence of school bullying in Korean middle school students. Arch Pediatr Adolesc Med 2004; 158:737-41.

27. Bond L, Wolfe S, Tollit M, Butler H, Patton G. A comparison of the Gatehouse Bullying Scale and the peer relations questionnaire for students in secondary school. J Sch Health 2007; 77:75-9.

28. Mynard H, Joseph S. Development of the Multidimensional PeerVictimisation Scale. Aggress Behav 2000; 26:169-78.

29. Arora CM, Thompson DA. My life in school checklist. In: Frederickson N, Cameron RJ, (series eds.), Sharp S (vol. eds.). Bullying Behaviour in Schools: Psychology in Education Portfolio. Windsor: NFER NELSON, 1999.

30. Schäfer M, Korn S, Smith PK, et al. Lonely in the crowd: Recollections of bullying. Br J Dev Psychology 2004; 22:379-94.

31. Thompson JK, Cattarin J, Fowler B, Fisher E. The Perception of Teasing Scale (POTS): a revision and extension of the Physical Appearance Related Teasing Scale (PARTS). J Pers Assess 1995; 65:146-57.

32. Orpinas PK. Skills training and social influences for violence prevention in middle schools: a curriculum evaluation (dissertation). Houston, TX: Texas Medical Center, 1993.

33. Parada RH. Adolescent Peer Relations Instrument: A theoretical and empirical basis for the measurement of participant roles in bullying and victimisation of adolescence: An interim test manual and a research monograph: A test manual. Bankstown: Publication Unit, Self-concept Enhancement and Learning Facilitation (SELF) Research Centre, University of Western Sydney, 2000.

34. Eisenberg ME, Neumark-Sztainer D, Perry CL. Peer harassment, school connectedness, and academic achievement. J Sch Health 2003; 73:311-6.

35. Gatignon H. Statistical Analysis of Management Data. New York, NY: Springer, 2010.

36. Schmitt TA. Current methodological considerations in exploratory and confirmatory factor analysis. J Psychoeduc Assess 2011; 29:304-21. 